\title{
THEORETICAL AND EXPERIMENTAL ANALYSIS OF TECHNICAL CONDITION OF PISTON RINGS OF VESSEL DIESEL INSTALLATIONS BY METHOD OF RESONANCE OSCILLATIONS
}

\author{
Aleksandrs Gasparjans ${ }^{1}$, Aleksandrs Terebkovs ${ }^{1}$, Anastasija Ziravecka ${ }^{2}$ \\ ${ }^{1}$ Latvian Maritime Academy, Latvia; ${ }^{2}$ Riga Technical University, Latvia \\ aleksandrs.gasparjans@latja.lv, sashater@bkc.lv, zhiravecka@eef.rtu.lv
}

\begin{abstract}
This work is a further continuation of the investigations presented before at the previous conference. The basic attention is turned to improvement of the accuracy of the measurements of the oscillation changing, to development of a specified mathematical model of the oscillating process. The idea of the method is to cause oscillations in the ring at the frequency of its own mechanical resonance and at the multiple harmonics. The amplitude of the ring oscillations is measured in the gap by means of sensors according to two coordinates simultaneously. The frequency, phase and amplitude of the oscillations depend on the mechanical properties of the ring's material and its geometry. Micro-cracks, the areas with stresses, hardening defects, local overheating, loss of elasticity and other defects cause deviations of frequency, phase and amplitude from the standard. One of the special features of the proposed method is registration of the frequency response of the piston ring oscillations simultaneously in two planes - radial (the plane of the ring) and in the direction of axis. It allows to discover the defects (microcracks, thermal inhomogeneity, etc.) of the ring independently on their orientation along the assumed axes. This is a kind of non-destructive methods.
\end{abstract}

Keywords: diagnostics, oscillations, frequency, amplitude, resonance, spectrum, harmonics.

\section{Introduction}

The suggested methodology allows an accurate detection of the current technical condition of the rings. The oscillations of the ring are researched in two conditions - under the condition of free and forced oscillations. Under the condition of free oscillations the ring experiences a disturbance form a single beat of a ball with a particular mass and angle of inclination. Under the condition of forced oscillations the undamped oscillations are excited in the ring at the frequency of its own mechanical resonance and at the multiple harmonics. Under this operation condition the amplitude, frequency and oscillation phase of the ring edges in the air gap are measured simultaneously along some co-ordinates by means of magneto-electric sensors. The accuracy of the oscillation parameter measurements was improved, more suitable methods of registration and mathematical processing of the measurements results were applied. An up-to-date mathematical model is now describing the parameters of different forms of oscillations of the piston rings of the internal combustion engine with an accepted accuracy.

Microcracks, heterogeneous stress-strain areas, hardening defects, local overheating, loss of elasticity and other failures cause deviations of frequency, phase and amplitude from the indicators accepted for the standards. An experimental bench is developed for the investigation of the technical condition of the vessel diesel piston ring of 500-720 mm diameter in a resonance regime. The described method of the technical diagnostics is topical for the determination of the current technical condition of the piston rings of high power diesel engines - vessel, gas compressing stations, electric stations - at their technical maintenance when the whole cylinder piston group is examined. The paper contains the results of these experiments. The results are compared with those of the mathematical modelling. In the first assumption the cross-section area can be considered rectangular. In fact, it has a complex form with declined oil grooves and holes. The calculation of its resonance frequency is a complex enough task. The first stage of the diagnostics is the comparison of the parameters of the oscillations of the left and right parts of the ring. The second stage is a comparison of the results with some etalon values. The etalon values are assumed the average results of the measurements of some valid piston rings. At the presence of several rings having already some defects discovered by other methods of non-destructive testing (fluoroscopic, ultrasound, luminescent, etc.) it is possible to elaborate a matrix of typical defects according to the results of these rings testing by means of the resonance method. The matrix of defects will provide an opportunity to find out not only a defect itself, but its character and location as well. A lot of fundamental literature is devoted to the theory of mechanical oscillations [1;2]. Analysis of the literature in the theory of cylindrical casings is presented in $[3 ; 4]$, in beams and plates in $[5 ; 6]$, in cylindrical closed rings in $[7 ; 8]$. However, the mathematical modelling of the oscillations and the processes in the split metal rings on the examples of the piston 
rings are not considered fully enough. This paper considers such questions directly. The basic purpose of it is to research the means of obtaining, registration and processing of the experimental data with their further analysis.

\section{Materials and methods}

An improved bench has been developed for investigation of the dynamic parameters of the piston ring oscillations. It gives an opportunity to study the parameters of the free damped and forced oscillations at the frequency of own mechanical resonance as well as its harmonics. The parameters to be registered are frequency, amplitude, time of oscillations damping, form of the oscillations, etc. A serviceable piston ring as well as damaged were examined.

At the beginning the mathematical model development required a simplified variant of the model. If the ring diameter is respectively large (the outer diameter is $512 \mathrm{~mm}$, its cross-section $16.5 \times 14.0$ $\mathrm{mm}$ ), some elastic plate can be conditionally distinguished from its full size (Fig. 1).

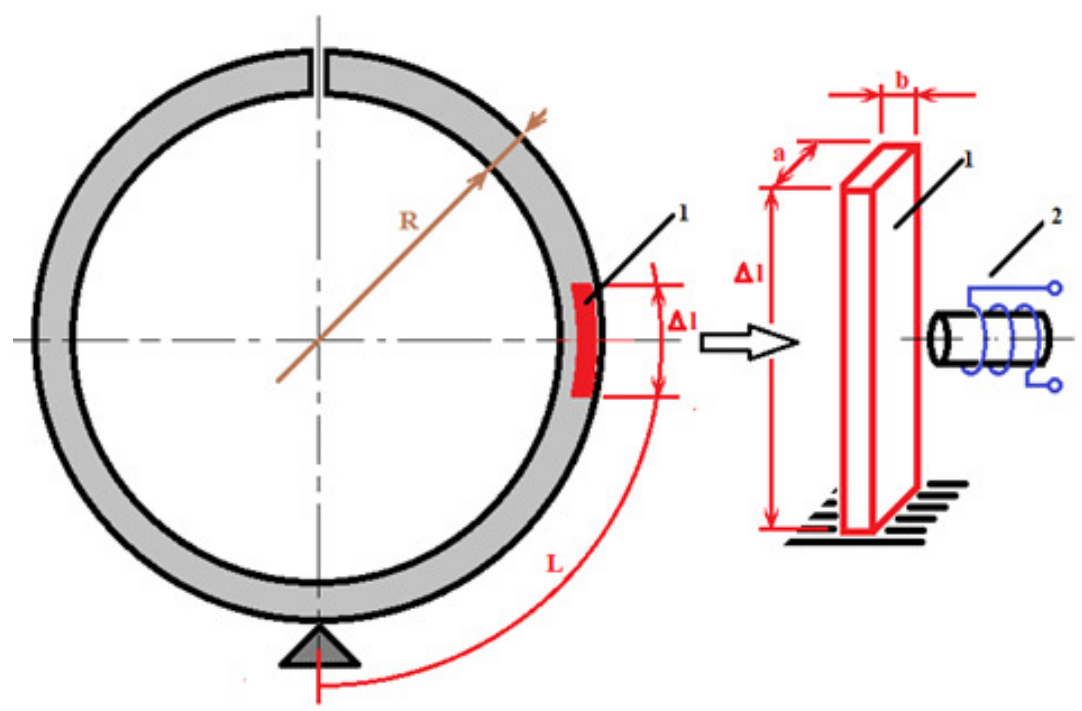

Fig. 1. Elastic plate (1) as fragment of piston ring, magnet-electric sensor of oscillation parameters (2)

The length of the plate $\Delta l$ is much less than the length of the circle segment $\mathrm{L}$. A metal plate close in its chemical composition and mechanical properties to the material of the piston ring has been selected for the experiments. The length of the plate 1 can be changed within the range from $100 \mathrm{~mm}$ to $450 \mathrm{~mm}$. The width of the plate is $2 \mathrm{~mm}$, that is about $16.5 / 2=1 / 8.25$ from the cross-section of the piston ring. The weight of the ring is $2.55 \mathrm{~kg}$. The oscillations of the plate were examined on the test bench presented in Fig. 2. An analogue signal from the magnet-electric sensor 2 comes to the digital double-channel oscilloscope 3. From the oscilloscope 3 the digital signal is supplied to the computer 4 . The obtained information is processed and memorized for further processing.

The results of an experimental study of the amplitude frequency response of free oscillations of a steel elastic beam clamped at one end and loaded with a certain mass from the other end are given in the article.

Fig. 2. shows an elastic beam of length $l$. The beam can deflect by an angle $\alpha$. In this case, the centre of gravity of the load moves to a distance $Y_{1}$, and rotates by an angle $Y_{2}$. Thus, this system has 2 degrees of freedom. In this case, $l>>Y_{1}$ and by the rotation of the centre of gravity of the shifted cargo can be neglected of $Y_{2}$. That is, it is possible to assume that the system has one degree of freedom. In the course of the experiments $Y_{1}$ is equal to $3 \mathrm{~mm}$, and the length of the beam $l$ varied and was equal to $60,90,120$ and $450 \mathrm{~mm}$.

Electromagnetic relays РКМП-2 contactless and without armature were applied as magnetoelectric sensors 2. The number of turns of the winding is 12100 , diameter of the wire is $0.11 \mathrm{~mm}$, the resistance of the winding is $1000 \Omega$. The double-channel digit oscilloscope 3-HAMEG Instruments 507 with control from a personal computer 4 . 


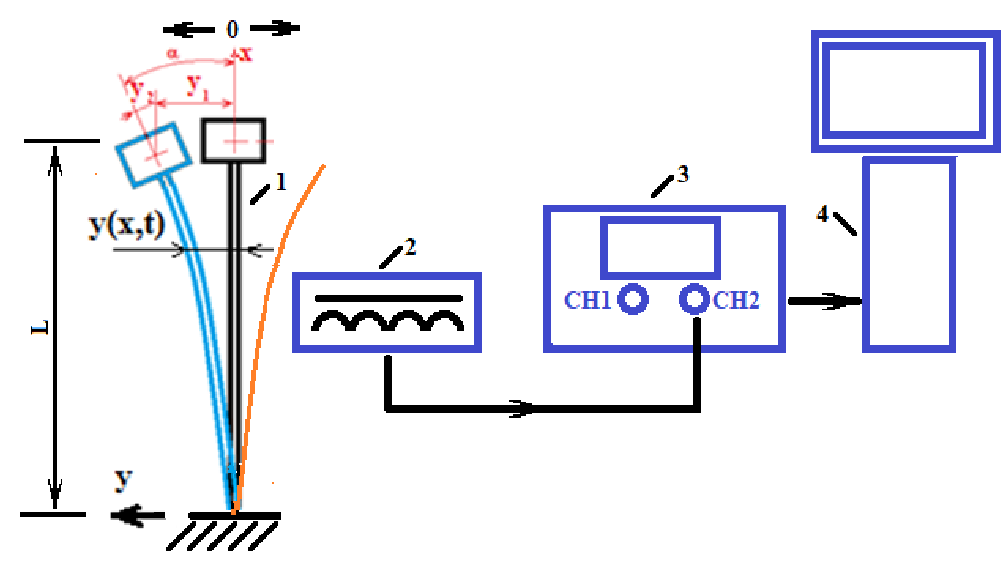

Fig. 2. Installation for investigation of elastic plate oscillations: 1 - elastic plate; 2 - magnetelectric sensor of oscillation parameters; 3 - digital oscilloscope 4 -computer with software

The end of the beam with the load deviated with a known force at $Y_{1}=3 \mathrm{~mm}$, then the force was suddenly removed. The system came into the mode of its own free damped vibrations.

Let us consider the own oscillations of the elastic plate described with the following differential equation [9-11]:

$$
\frac{\partial^{2} y}{\partial t^{2}}+k_{1}^{2} \frac{\partial^{4} y}{\partial x^{4}}=0
$$

where $E$ - modulus of elasticity, Pa;

$J$ - moment of inertia of the cross-section of the plate, $m^{4}$;

$m$ - linear weight, $\mathrm{kg}$;

$$
k_{1}^{2}=\frac{E J}{m} .
$$

The partial solution for the basic oscillation is in the following way $[9 ; 10]$ :

$$
y(x, t)=\varphi(x) \sin (p t+\alpha) .
$$

Differentiate twice in time:

$$
\frac{\partial^{2} y}{\partial t^{2}}=-p^{2} \varphi(x) \sin (p t+\alpha) .
$$

And four times in coordinate:

$$
\frac{\partial^{4} y}{\partial x^{4}}=\frac{\partial^{4} \varphi}{\partial x^{4}} \sin (p t+\alpha)
$$

After the applying of (2) and (3) in (1), we will get

$$
\frac{\partial^{4} \varphi}{\partial x^{4}}-k_{2}^{4} \varphi(x)=0
$$

where

$$
k_{2}^{4}=\frac{m p^{2}}{E J} .
$$

Equation (4) has four independent partial solutions:

$$
\varphi_{1}=\cos k x, \varphi_{2}=\sin k x, \varphi_{3}=\operatorname{ch} k x, \varphi_{4}=\operatorname{sh} k x .
$$

As equation (4) is linear, its common solution is a linear combination of the partial solutions: 


$$
(x)=D_{1} \cos k x+D_{2} \sin k x+D_{3} \operatorname{ch} k x+D_{4} \operatorname{sh} k x,
$$

where

$$
\operatorname{ch} k x=\frac{e^{k x}+e^{-k x}}{2} ; \operatorname{sh} k x=\frac{e^{k x}-e^{-k x}}{2}-\text { hyperbolic functions. }
$$

The variables are taken from the boundary conditions.

The boundary conditions at $x=0$ :

$$
\varphi(0)=0 ; \frac{(0)}{x}=0 .
$$

Let us fix two boundary conditions at $x=L$ : the first one corresponds to zero value of the torque acting on the free end

$$
\frac{\partial^{2}(L)}{\partial x^{2}}=0,
$$

the second conditionis adifferential equation of movement of the mass concentratedon the end of the plate

$$
E J \frac{\partial^{3}(L)}{\partial x^{3}}=-m p^{2} \varphi(L)
$$

It is called a boundary task with non-integrated boundary conditions [10]. The mathematical tool presented above has been modelled in MATLAB environment.

Fig. 3 represents the results of the mathematical modelling of the plate oscillations at particular geometry and physical parameters (all the parameters are in relative units). Thus, the result is a sample for comparison with the presented experimental data of the plate oscillations.

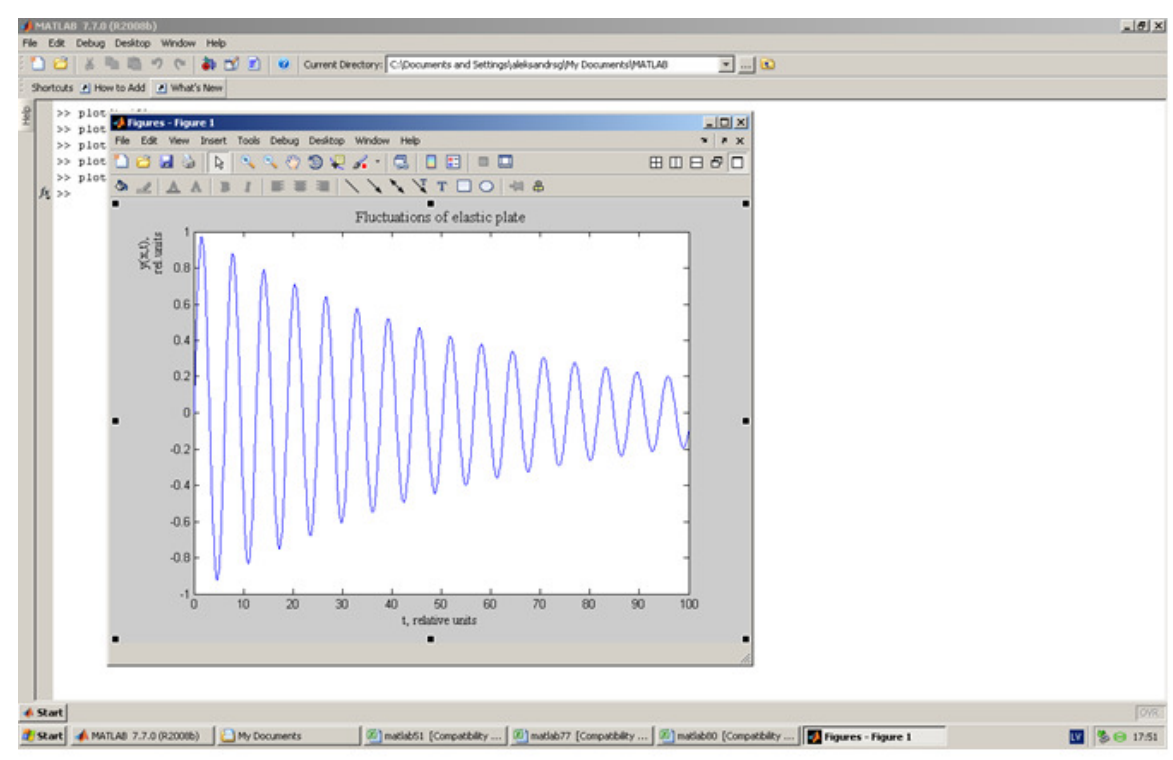

Fig. 3. Result of mathematical modelling for sample plate

The frequency of the natural vibrations can be determined using a formula (1): [1;3;12]:

$$
f=\frac{1}{2 \pi} \frac{\alpha^{2}}{l^{2}} \sqrt{\frac{E J}{m}}, \mathrm{~Hz},
$$

where $l$-beam length, m;

$E$ - elasticity of material, $\mathrm{Pa}$;

$J$ - beam flywheel action, $\mathrm{m}^{4}$; 
$\alpha$ - root of transcendental equation of bending vibration frequency; for the mentioned beam bonding technique $\alpha=1.875$;

$m$ - given mass per unit length of the beam.

The mass can be determined using a formula:

$$
m=m_{0}+\frac{1}{l} \sum_{1}^{n} k_{n} m_{n}
$$

where $m_{0}$ - fairly spread mass;

$m_{n}$ - concentrated mass;

$n$ - number of concentrated mass;

$k_{n}$ - reduction coefficient of concentrated mass to uniformly distributed mass depending on the type of beam supporting.

Flywheel action for $\mathbf{J}$ for a rectangular beam is equal to:

$$
J=\frac{b h^{3}}{12}
$$

where $b$-siding of beam, $\mathrm{m}$;

$h$ - beam depth (thickness), m.

The following fact should be taken into account that the elastic plate 1 can twist not only along its length, but can also have some nodes and antinodes, like it is in Fig.4. b. In this case the node is located at point A. Additionally, the second harmonic of the basic frequency appears in the harmonics spectrum. It is visible in the spectrogram in Fig. 5. With decreasing of the oscillations amplitude in the damping process, the number of the nodes and, as a result, the number of harmonics are also decreasing. At the lower amplitudes of the oscillations the form of the plate's curving becomes close to that demonstrated in Fig. 4.a. The forms of the elastic oscillations of the piston ring are considered and analysed in [12]. Fig.5. represents the experimental results of the spectrum of the elastic plate oscillations depending on its length $L$. The plate with the defect $\mathrm{S}$ was also examined for the analysis of oscillations. The circuit of the test is shown in Fig. 6. One magnet-electric sensor of oscillations 2a was placed on the top of the plate above the place of the defect $\mathrm{S}$. The second similar sensor $2 \mathrm{~b}$ was placed under this place. This gives an opportunity to control the oscillation parameters on both sides of the plate. Both oscillation sensors were connected to two inputs $\mathrm{CHI}$ and $\mathrm{CHII}$ of the digital oscilloscope 3 (Fig.2).

The plate was gradually forcibly damaged with the cut $\mathrm{S}$ with the width $2,4,8$ and $10 \mathrm{~mm}$, that is $7.1 \%, 14.4 \%, 28.6 \%$ and $35.7 \%$ respectively of the width of the plate. The spectrum of the oscillations of the plate with the defect $S=10 \mathrm{~mm}$ is given in Fig. 7. The red curve corresponds to the signal from the upper sensor 2.a - oscilloscope input CHI, the blue one, if the signal is from the lower sensor 2.1 - CHII input.

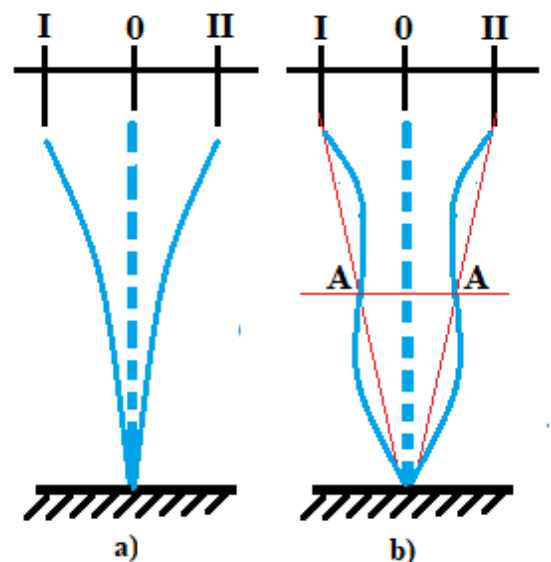

Fig. 4. Form of elastic plate oscillations 
Fig. 8 demonstrates the data of the spectral analysis of the oscillations of the plate with the length $L=390 \mathrm{~mm}$, with the width of the defect $0 \mathrm{~mm}, 2 \mathrm{~mm}, 4 \mathrm{~mm}, 8 \mathrm{~mm}$, and $10 \mathrm{~mm}$. With increasing of the defect width new harmonics appear with the frequencies of about121, 148, 179, 201, $300 \mathrm{~Hz}$ (harmonics f26, f32, f38, f 42, f64).Harmonics of the highest frequencies are not shown in Fig. 8.

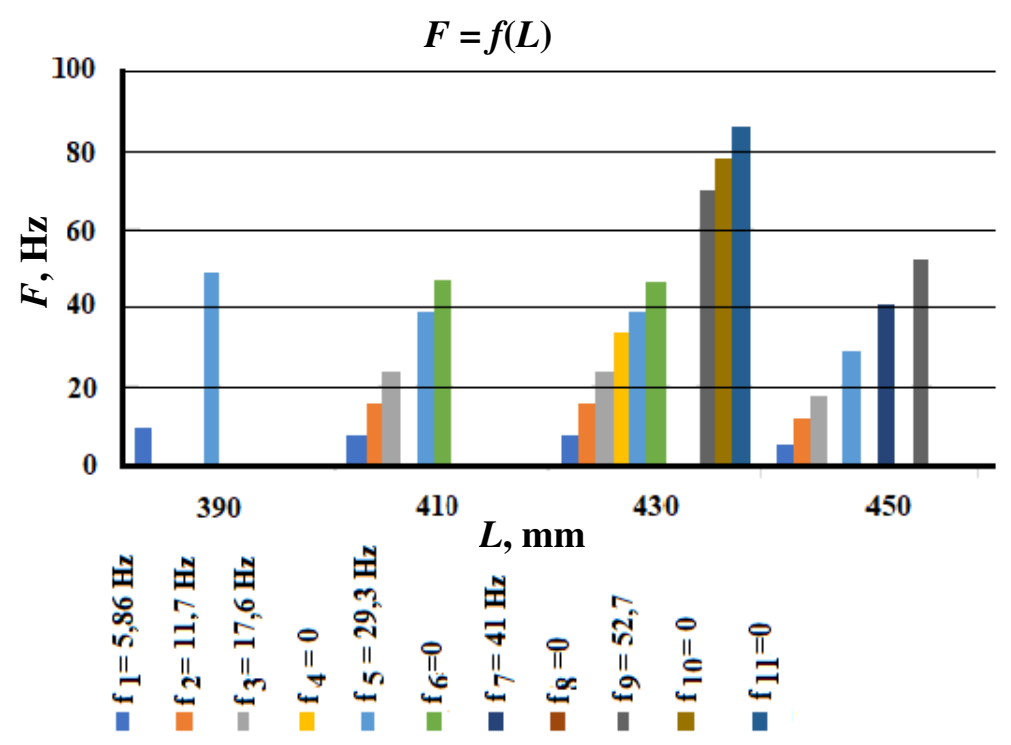

Fig. 5. Frequency spectrum of plate oscillations depending on its length $L$

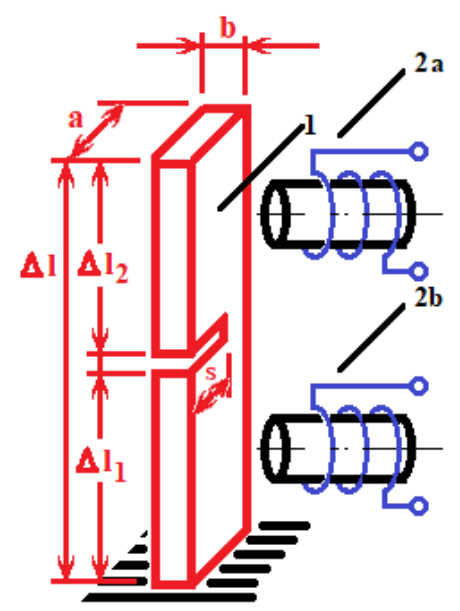

Fig. 6. Scheme of frequency

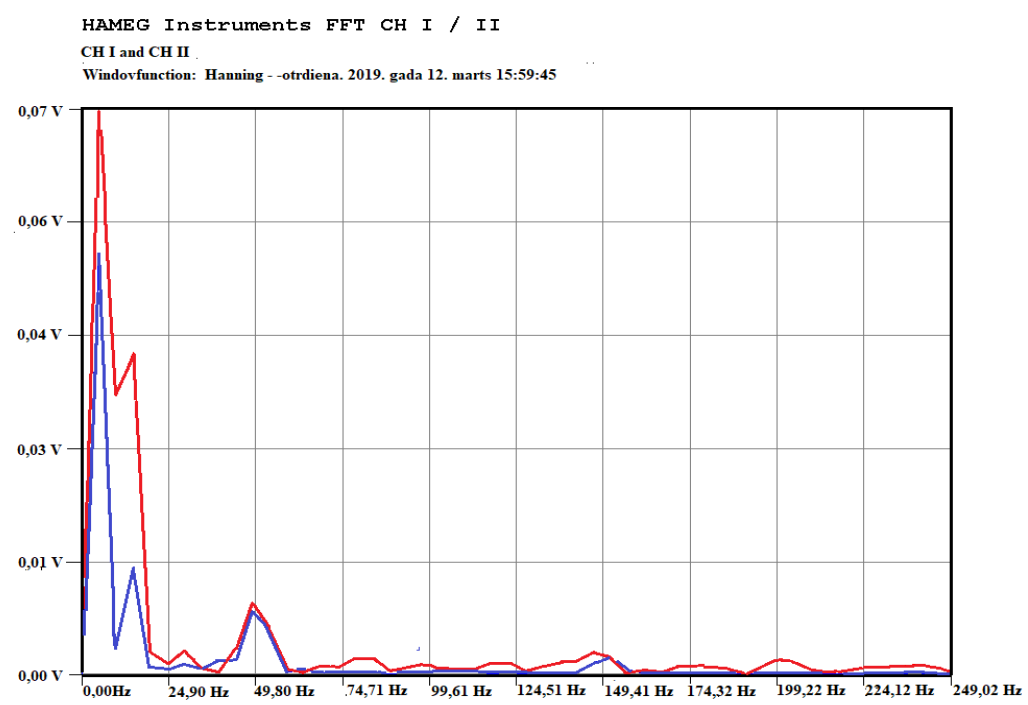

Fig.7. Frequency spectrum of plate with defect characteristics for plate with defect $L=390 \mathrm{~mm}, S=10 \mathrm{~mm}$

$$
F=f(S)
$$

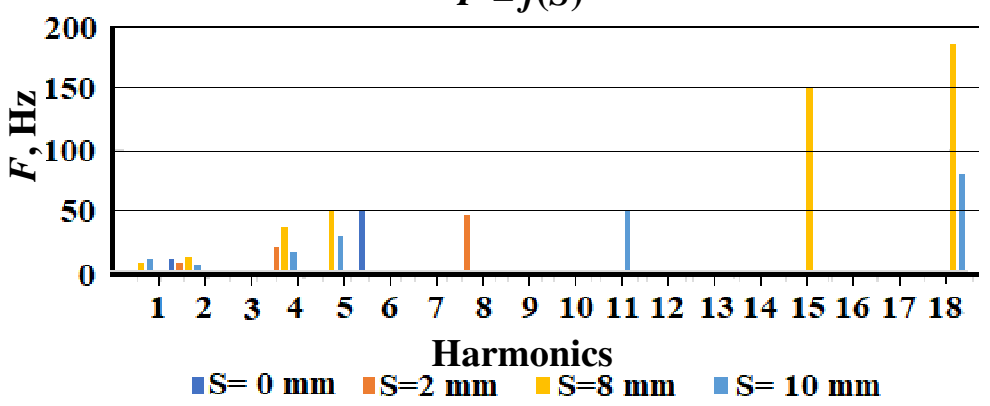

Fig. 8.Spectrum of oscillations of plate with length $L=390 \mathrm{~mm}$ with defects $S$ width $0,2,4,8$ and $10 \mathrm{~mm}$ 


\section{Results and discussion}

1. Appearance of the harmonics with the frequency that exceeds the basic one 5 times or more signals about the existence of defects in the sample.

2. The described method to monitor the mechanical properties of the piston ring material can be applied with the input monitoring of the piston rings. In most of the cases the traditional methods use the measurements of the ring geometry in closed and free condition, mass of the ring, gaps only.

3. Application of the method of amplitude-frequency response allows discover the presence of microcracks, areas of different types of thermal inhomogeneity and elasticity.

4. The experimental data (frequencies of harmonics and their amplitude) coincide with the modelling results. The accuracy is 10 to $12 \%$; that is admissible at the stage of the preliminary investigations.

\section{Conclusions}

The spectral characteristics of the oscillations give an opportunity to judge about the presence of defects in the sample.

The comparison of the etalon sample or its mathematical model (amplitude, phase, form, time of oscillation damping, etc.) with the sample under the experiments allows a precise enough determination of the place of the defect.

The method can be useful at the input and current control and monitoring of the technical condition of piston rings. In practice about 3-5\% of newly installed piston rings are broken during the first 100-300 motor-hours. As usual the defect is detected in the area of the lock of the piston ring.

Unlike the well-known methods of the amplitude-frequency response measurement, the authors of this paper measure simultaneously along two coordinates: in the plane of the ring - the radial oscillations; and in the axial plane - perpendicular to the plane of the ring.

The suggested method of the piston ring condition monitoring refers to the non-destructive methods.

\section{Further planned work}

The method of detection of the current technical condition of piston rings is planned to be implemented at Riga Shipyard to test it under the factory conditions.

\section{References}

[1] Graham K.S. Fundamentals of Mechanical Vibrations.Paperback- International Second Edition, 2000. $630 \mathrm{pp}$.

[2] Biderman I.L. Theory of mechanical oscillations. M.: Vischaja shkola, 1980. 408 p. (In Russian).

[3] Naeem M.N., Kanwal S. Vibration Characteristics of Ring-Stiffened Functionally Graded Circular Cylindrical Shells. ISRN Mechanical Engineering. Volume 2012 (2012), 13 p.

[4] Loy C. T., Lam K. Y., Reddy J. N. Vibration of functionally graded cylindrical shells. International Journal of Mechanical Sciences, vol. 41, no. 3, 1999, pp. 309-324.

[5] Benachour A., Tahar H. D., Atmane H., A.Tounsi., A., Ahmed M. S. A four variable refined plate theory for free vibrations of functionally graded plates with arbitrary gradient. Composites Part B, vol. 42, no. 6, 2011, pp. 1386-1394.

[6] Benyoucef S., Mechab I., Tounsi A., Fekrar A., Adda Bedia E. A. Bending of thick functionally graded plates resting on Winkler-Pasternak elastic foundations. Mechanics of Composite Materials, vol. 46, no. 4, 2010, pp. 425-434.

[7] Chidamparam P., Leissa A. W.Vibrations of Planar Curved Beams, Rings, and Arches. ASME theAmerican Society of Mechanical Engineering. Appl. Mech. Rev 46(9), 2009, pp. 467-483.

[8] Naeem M. N., Arshad S. H., Sharma C. B. The Ritz formulation applied to the study of the vibrationfrequency characteristics of functionally graded circular cylindrical shells. Proceedings of the Institution of Mechanical Engineers Part C, vol. 224, no. 1, 2010, pp. 43-54.

[9] Kosicin A.V. Method of vibrodiagnostics of the elastic constructions on the basis of own oscillations analysis, № 2 (3), 2011. Navy Academy of the Republic of Belarus. pp. 129-135. (In Russian). 
[10] Babakov I.F. Fluctuation theory. M: Drofa. 2004. 591 p. (In Russian).

[11] Grigoriev A. Matkin Uj. Modelling of fluctuations of elastic plate of the phematic vibrator. Tula Technical universitate.2010 2. path 2. 86 - 92 pp. (In Russian).

[12] Gasparjans A., Terebkovs A., Ziravecka A. Application of resonance method for examining of piston ring technical condition // 16th International Scientific Conference,University of Agriculture Faculty of Engineering.Engineering for Rural Development. Proceedings, Volume 16, ISSN 1691-5976, May 24-26, 2017, Jelgava, Latvia. pp. 884-891. 